\title{
Application of a novel self-assembling peptide to prevent hemorrhage after EMR, a feasibility and safety study
}

\author{
Elsa Soons $^{1} \cdot$ Ayla Turan $^{1}$ (D) Erwin van Geenen ${ }^{1} \cdot$ Peter Siersema $^{1}$
}

Received: 10 April 2020 / Accepted: 10 July 2020 / Published online: 17 August 2020

(c) The Author(s) 2020

\begin{abstract}
Background A novel self-assembling peptide (SAP) can be applied to the post-endoscopic mucosal resection (EMR) defect to treat oozing bleedings. It has been suggested to stimulate early healing of damaged vessels. We hypothesized that SAP application could prevent delayed bleeding (DB) after EMR and performed a prospective cohort study to determine feasibility and safety.

Methods A total of 48 consecutive patients who underwent EMR between June 2018 and August 2019 for large lesions in the esophagus, duodenum $(>1 \mathrm{~cm})$ or colorectum $(>2 \mathrm{~cm})$ were treated with adjuvant SAP application. Duration and ease of SAP application were measured, as well as DB outcome.

Results The EMR defects of 48 patients were treated with SAP; 17 in the esophagus, 13 in the duodenum and 18 in the colorectum. SAP was easy to apply on the EMR defect with a median duration of $2.0 \mathrm{~min}$. A dose of $3 \mathrm{cc}$ was generally enough to cover a defect between 10 and $50 \mathrm{~mm}$. An exploratory analysis of the prophylactic ability of SAP showed that $15.9 \%$ of patients (7/44) treated with SAP still had a DB, mostly in the duodenum (4/11). No adverse events related to gel exposure were reported.

Conclusions SAP application after EMR was found to be feasible and safe, and did not delay the procedure; however, DB was still relatively common. Future comparative studies are needed to evaluate whether SAP is able to reduce DB after EMR, particularly for lesions with an increased bleeding risk, such as in the duodenum.
\end{abstract}

Keywords Endoscopic mucosal resection $\cdot$ Topical agent $\cdot$ Prophylaxis $\cdot$ Feasibility $\cdot$ Safety $\cdot$ Delayed bleeding

Endoscopic mucosal resection (EMR) is a curative treatment option for dysplastic lesions and early cancers in the gastrointestinal tract. Although EMR is generally a safe procedure, adverse events still occur with delayed bleeding (DB) being the most common. DB rates after EMR have been reported between 1 and $2 \%$ in the esophagus [1-3], $0-28 \%$ in the duodenum $[4,5]$ and $3-12 \%$ in the colorectum [6].

Elsa Soons and Ayla Turan contributed equally to this project.

Electronic supplementary material The online version of this article (https://doi.org/10.1007/s00464-020-07819-7) contains supplementary material, which is available to authorized users.

Ayla Turan

ayla.turan@radboudumc.nl

1 Department of Gastroenterology and Hepatology, Radboud University Medical Center, Geert Grooteplein Zuid 10, 6500 HB Nijmegen, The Netherlands
Various methods have been proposed to decrease the risk of DB after EMR, such as snare tip soft coagulation of visible vessels or prophylactic clipping of the post-EMR defect. However, the benefit of these measures is still not sufficiently clear [7-10]. In the case of prophylactic clipping, the efficacy is highly dependent on achieving complete closure, which, even in experienced hands and with an adequate technique, can be difficult to achieve (e.g. when the post-EMR defect is large or located over a fold) [11]. A novel simple technique to promote hemostasis and wound regeneration is to cover the defect with a self-assembling peptide (PuraStat ${ }^{\circledR}, 3-D$ Matrix Europe, France,SAP). The matrix that is formed after application of SAP has been hypothesized to prevent DB after EMR [12]. To date, studies on SAP have mostly focused on endoscopic submucosal dissection, and less is known about the effect of SAP after EMR [12-14]. Our aim was to perform a prospective study on safety and feasibility of prophylactic SAP application after EMR. In addition, we evaluated DB rate after SAP application. 


\section{Materials and methods}

A single-center prospective cohort study was conducted at a tertiary referral center in Nijmegen, the Netherlands. Forty-eight consecutive patients who underwent EMR of the esophagus, duodenum or colorectum between June 2018 and August 2019 were treated with SAP. All EMR-procedures were performed by experienced endoscopists who each had performed $>300$ previous endoscopic resections. Submucosal fluid injection in duodenal and colorectal EMR was performed with hydroxyethyl starch solution (Voluven, Fresenius Kabi, the Netherlands) colored with indigo carmine. Adrenaline was not added routinely. Standard generator settings for large polypectomies were used for all EMRs in the esophagus and colorectum (VIO 200 D; EndoCUT Q mode, Effect 3, Cut distance 1, Cut interval 6; Erbe Elektromedizin $\mathrm{GmbH}$, Tübingen, Germany) and in the duodenum (VIO 200 D; EndoCUT Q mode, Effect 2, Cut distance 1, Cut interval 6). Snare tip coagulation after esophageal and colorectal EMR was performed with the settings Forced Coag, Effect 2, $60 \mathrm{~W}$, and after duodenal EMR with Forced Coag, Effect 2, $\max 20 \mathrm{~W}$. This study was approved by the Medical Ethics Review Committee of the Radboudumc, Nijmegen (reference number: 2018-4392) and was carried out in accordance with the Helsinki Declaration. The study has been registered at the Dutch Trial Register (reference number: NTR7338).

\section{Participants}

Patients older than 18 years referred for EMR of the esophagus, duodenum or colorectum were screened. They were invited to participate when the diameter of the lesion was estimated to be $\geq 10 \mathrm{~mm}$ for esophageal or duodenal lesions, or $\geq 20 \mathrm{~mm}$ for colorectal lesions. All patients gave informed consent before initiation of the EMR-procedure.

\section{Materials and procedure}

SAP is a transparent gel containing inactivated synthetic peptides. When these peptides come in contact with blood or tissue fluid they are $\mathrm{pH}$-activated, forming a three-dimensional scaffold structure of nanofibers. This is proposed to result in a physical barrier that stops small bleedings. Furthermore, the nucleotides in the gel are supposed to promote wound regeneration [15]. The gel is applied via a dedicated catheter that is inserted through the working channel of an upper or lower endoscope (PuraStat Nozzle System type E, Top Corporation, Tokyo, Japan). After the EMR procedure, an adequate volume of SAP was applied to cover the total surface of the EMR-induced ulcer, using a 3- or $5 \mathrm{cc}$ syringe. Endoscopists were instructed to desufflate air after the application of SAP. By doing this, the intestinal wall will fall back into its original state and the folds will potentially prevent migration of the gel. The decision to use other prophylactic measures (e.g. clip placement) was left to the treating physician, as current guidelines are not conclusive on this subject [16].

\section{Study design}

All patients who were treated with SAP were prospectively followed for 30 days after the procedure. Data were collected in Castor EDC (Castor Electronic Data Capture, Ciwit BV, Amsterdam, the Netherlands), an online Electronic Data Capture platform.

\section{Outcomes}

Primary endpoint was feasibility of SAP application, including volume used per $\mathrm{cm}^{2}$ of resection surface, EMR procedure time, duration of gel application and safety. Secondary outcomes included DB within 30 days post-procedure, with exclusion of patients who had undergone other prophylactic measures (e.g. clip placement), and defined as hematemesis or rectal blood loss requiring emergency room visit, blood transfusion, prolongation of hospital stay, re-hospitalization or endoscopic, radiologic or surgical intervention [17-20]. The severity of DB was described according to the ASGE guidelines $[17,19]$ as mild, moderate, severe or fatal. Risk of DB was evaluated according to patient related risk factors, such as location and size of the lesion, cardiovascular disease and anticoagulant use [18, 21, 22].

\section{Statistical analysis}

Categorical data are presented as counts and percentages and compared using the $\chi^{2}$ test where applicable. Continuous data are reported as means \pm standard deviation (SD) when normally distributed, or as median with the interquartile range (IQR) when not normally distributed and compared using the Student's $T$ test where applicable. For the interpretation of DBs, data were divided into three groupsesophageal, duodenal and colorectal lesions-because DBrisk between different locations in the gastrointestinal (GI) tract is known to vary [1-6]. All analyses were done in IBM SPSS Statistics, version 25 (SPSS Inc., Chicago, IL, USA).

\section{Results}

\section{Baseline characteristics}

Eighty-two patients were screened for eligibility, of which 48 patients, subdivided for location in the esophagus, 
duodenum and colorectum, were included and treated with SAP. Four patients were excluded from the secondary analysis due to intraprocedural clip placement. The flow diagram of inclusion is presented in Fig. 1.

A total of 17, 13 and 18 patients underwent EMR of the esophagus, duodenum and colorectum, respectively. As can be seen in Table 1, the median age was 68.5 years and $60.4 \%$ were men. Most patients were graded as ASA (American Society of Anesthesiologists physical status classification) grade $2(64.6 \%)$. A total of $22.9 \%$ of patients was smoker and $41.7 \%$ regularly used alcohol. Half of patients had cardiovascular comorbidity, mostly hypertension (20.8\%). Thirty-five percent of patients used antithrombotic medication, typically a platelet inhibitor (16.7\%). Antithrombotic medication was managed according to the 2016 guideline from the Dutch association of gastroenterologists and hepatologists (NVMDL), including that platelet inhibitors were generally continued and in case of double therapy clopidogrel was stopped, Vitamin $\mathrm{K}$ antagonists were stopped five days prior to the EMR or bridged with low-molecular-weight heparin (LMWH), and direct oral anticoagulants (DOACS) were stopped two to three days before the EMR. All antithrombotic drugs were restarted the following day, unless decided otherwise by the treating endoscopist. None of the included patients was known with portal hypertension.

\section{Primary outcome: feasibility of application}

The application of the gel to the wound surface was performed systematically from the distal to the proximal side of the defect (see Video 1). SAP application was overall experienced as easy. Nonetheless, due to the transparency of the gel, it was sometimes considered difficult to confirm that the total lesion surface was completely covered (see Fig. 2). Also, the position of the EMR defect, and as a result the effect of gravity, was sometimes found to impede SAP application, which required covering some areas repeatedly (See Video 2). The median volume per $\mathrm{cm}^{2}$ was $0.6 \mathrm{cc}$ (IQR $0.3-1.2$ ), applied in a median time of $2.0 \mathrm{~min}$ (IQR 1.0-2.5) (see Table 2).

Fig. 1 Flowchart of inclusion. EMR endoscopic mucosal resection, $I P$ intraprocedural, $I C$ informed consent

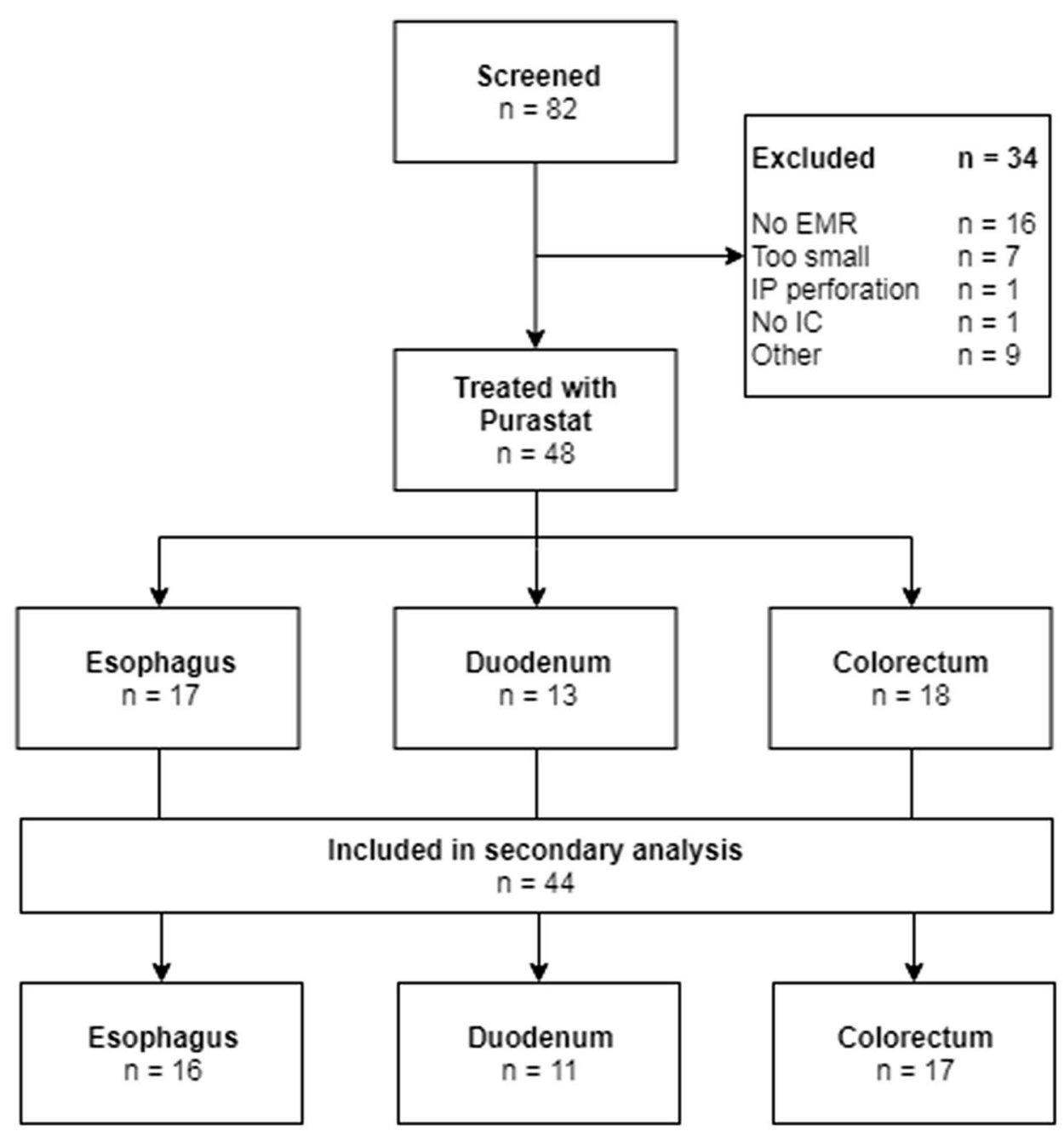


Table 1 Baseline characteristics

\begin{tabular}{|c|c|c|c|c|}
\hline & Overall $N=48$ & Esophagus $N=17$ & Duodenum $N=13$ & Colorectum $N=18$ \\
\hline Age in years, median (IQR) & $68.5(55.3-73.0)$ & $70.0(61.0-74.5)$ & $53.0(46.0-71.0)$ & $69.5(62.0-73.3)$ \\
\hline Sex (male), $n(\%)$ & $29(60.4)$ & $12(70.6)$ & $8(61.5)$ & $9(50.0)$ \\
\hline BMI, mean (SD) & $26.9(3.8)$ & $26.7(3.6)$ & $28.4(3.8)$ & $25.8(3.9)$ \\
\hline \multicolumn{5}{|l|}{ ASA, $n(\%)$} \\
\hline 1 & $3(6.3)$ & - & $2(15.4)$ & $1(5.6)$ \\
\hline 2 & $31(64.6)$ & $10(58.8)$ & $9(69.2)$ & $12(66.7)$ \\
\hline 3 & $12(25.0)$ & $6(35.3)$ & $1(7.7)$ & $5(27.8)$ \\
\hline 4 & $2(4.2)$ & $1(5.9)$ & $1(7.7)$ & - \\
\hline Smoking, $n(\%)$ & $11(22.9)$ & $5(29.4)$ & - & $6(33.3)$ \\
\hline Regular alcohol intake, $n(\%)$ & $20(41.7)$ & $11(64.7)$ & $1(7.7)$ & $8(44.4)$ \\
\hline Cardiovascular comorbidity, $n(\%)$ & $24(50.0)$ & $13(76.5)$ & $4(30.8)$ & $7(38.9)$ \\
\hline Hypertension & $10(20.8)$ & $6(35.3)$ & $1(7.7)$ & $3(16.7)$ \\
\hline \multicolumn{5}{|l|}{ Antithrombotic medication, $n(\%)$} \\
\hline No & $31(64.6)$ & $10(58.8)$ & $11(84.6)$ & $10(55.6)$ \\
\hline Platelet inhibitors & $8(16.7)$ & $4(23.5)$ & $1(7.7)$ & $3(16.7)$ \\
\hline Vit $\mathrm{K}$ antagonists & $6(12.5)$ & $2(11.8)$ & $1(7.7)$ & $3(16.7)$ \\
\hline Combination therapy ${ }^{a}$ & $1(2.1)$ & $1(5.9)$ & - & - \\
\hline DOAC/NOAC & $2(4.2)$ & 0 & - & $2(11.1)$ \\
\hline Portal hypertension, $n(\%)$ & - & - & - & - \\
\hline
\end{tabular}

$I Q R$ interquartile range, $B M I$ body mass index, $S D$ standard deviation, ASA America Society of Anesthesiologists, Vit $K$ antagonists vitamin $\mathrm{K}$ antagonists, $D O A C / N O A C$ direct oral anticoagulants/new oral anticoagulants

${ }^{\mathrm{a}}$ Combination therapy defined as concurrent use of aspirin and acenocoumarin

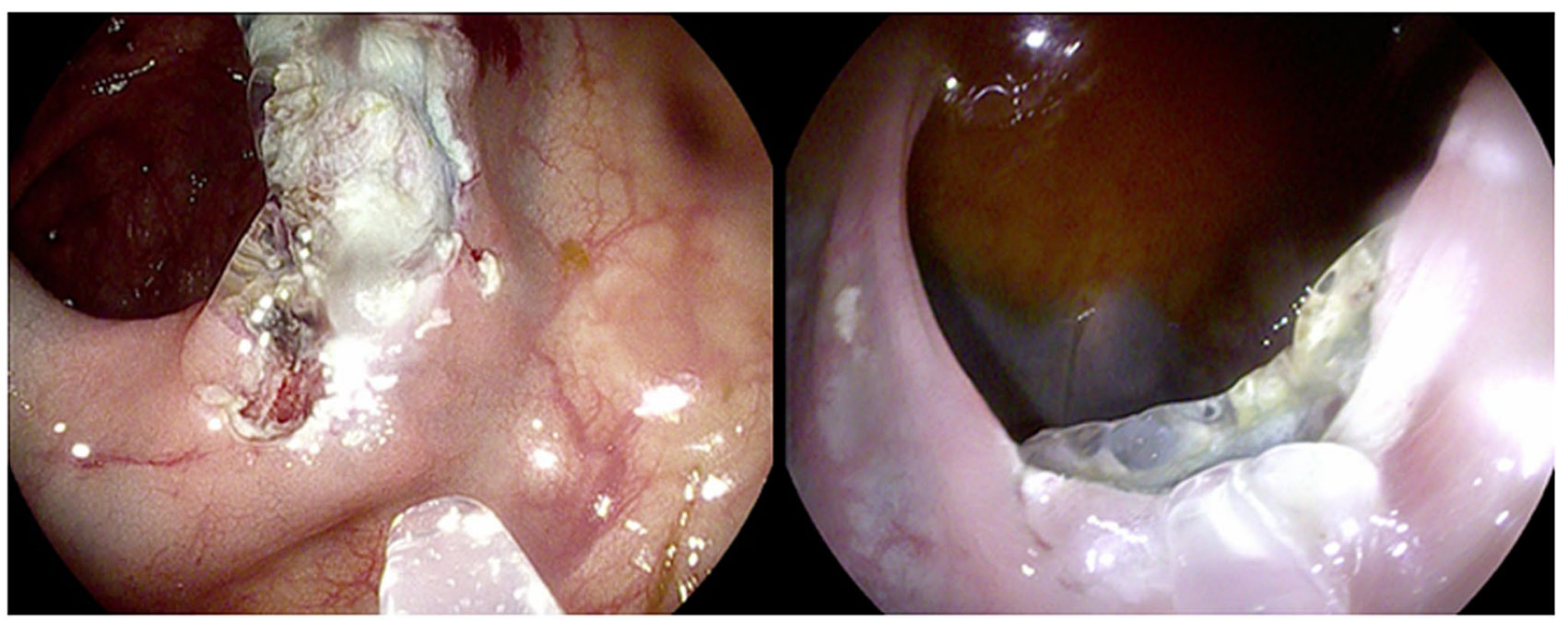

Fig. 2 Transparent SAP applied to two separate EMR defects with a catheter

Table 2 Specifications of SAP use

\begin{tabular}{ll}
\hline Volume SAP in cc, median (IQR) & $3.0(3.0-5.0)$ \\
Volume SAP per $\mathrm{cm}^{2}$ in cc, median (IQR) & $0.6(0.3-1.2)$ \\
Time to apply SAP in minutes, median (IQR) & $2.0(1.0-2.5)$ \\
Procedure time (minutes), median (IQR) & $60.0(45.0-86.8)$
\end{tabular}

$S A P$ self-assembling peptide, $I Q R$ interquartile range

\section{Safety}

No adverse events related to gel exposure, including allergy were reported. 


\section{Secondary outcome: delayed bleeding}

A total of 44 patients were included in the secondary outcome analysis. DB occurred in seven patients; one (6.3\%) in the esophagus, four (36.4\%) in the duodenum and two $(11.8 \%)$ in the colorectum. Three of the patients $(3 / 7,42.9 \%)$ with DB used antithrombotics (see Table 3). All patients with DB underwent a piecemeal EMR.

To assess the a priori risk of DB based on known patient related risk factors for bleeding, these risk factors were compared between patients with and without DB. The median age of patients with DB was 68 years and in those without 73 years. Although cardiovascular comorbidity, mostly hypertension, was more frequently present in the non-DB group (51.4\% vs. $42.9 \%$ ), platelet inhibitors were more frequently used in the DB group. Both DBs in the colorectal EMR group originated from a rectal (left sided) lesion. In addition, median size of colorectal lesions was $4 \mathrm{~cm}^{2}$ (2.0-12.5) in patients without DB and $25.5 \mathrm{~cm}^{2}(15-\infty)$ in patients with DB (see Table 3).

Patients with DB presented after a median of one day (IQR 0-11) and were admitted for a median of two days (IQR 1-6), with no need for ICU admission. All patients with DB underwent additional endoscopy, with six of seven patients being treated with clip placement for active bleeding $(85.7 \%)$. In one patient, clip closure was not successful because of intraprocedural clip detachment. Hemostasis of this rectal lesion was achieved after placement of two cross stich sutures and insertion of a hemostatic gelatin sponge tampon (Spongostan ${ }^{\circledR}$ ). During his hospital stay, the patient received two blood transfusions. Hereafter, he recovered quickly and could be discharged within two days. In one patient with previous EMR of the duodenum, second endoscopy was required due to continued blood loss. Treatment was performed with adrenaline injection, clip placement and bipolar coagulation (Gold probe; Boston Scientific, Marlborough, MA), and no recurrent bleeding occurred. In four patients $(57.1 \%)$, blood transfusion was required, with a median of two units (IQR 1.3-3.5). Most of the DBs (57.1\%, see Supplementary Tables 1 and 2) were classified as moderately severe according to the ASGE lexicon for endoscopic adverse events [19].

DBs classified as mild in this cohort, were seen in patients not using antithrombotics (see Supplementary Table 3). Nonetheless, all severe and half (2/4) of the moderately severe DBs were seen in patients using antithrombotic medication prior to the EMR. One patient, who used antiplatelet therapy, stopped his medication four days prior
Table 3 Patient related risk factors for bleeding in patients with and without DB

\begin{tabular}{|c|c|c|c|}
\hline & EMR without DB $N=37$ & EMR with DB $N=7$ & $P$ value \\
\hline Age in years, median (IQR) & $68.0(57.0-72.5)$ & $73.0(54.0-80.0)$ & NS \\
\hline Cardiovascular comorbidity, $n(\%)$ & $19(51.4)$ & $3(42.9)$ & NS \\
\hline Anticoagulant use, $n(\%)$ & & & NS \\
\hline No & $25(67.6)$ & $4(57.1)$ & \\
\hline Antiplatelet & $6(16.2)$ & $2(28.6)$ & \\
\hline Vit $\mathrm{K}$ antagonists & $4(10.8)$ & $1(14.3)$ & \\
\hline Combination therapy $^{\mathrm{a}}$ & $1(2.7)$ & - & \\
\hline DOAC/NOAC & $1(2.7)$ & - & \\
\hline Lesion location, $n(\%)$ & & & NS \\
\hline Esophagus & $15(40.5)$ & $1(14.3)$ & \\
\hline Duodenum & $7(18.9)$ & $4(57.1)$ & \\
\hline Colorectum & $15(40.5)$ & $2(28.6)$ & \\
\hline Specified location colorectum, $n(\%)$ & & & NS \\
\hline Right sided & $7(46.7)$ & - & \\
\hline Left sided & $8(53.3)$ & $2(100)$ & \\
\hline Lesion size in $\mathrm{cm}^{2}$, median (IQR) & & & 0.04 \\
\hline Esophagus & $3.5(2-6)$ & $4(4-4)$ & \\
\hline Duodenum & $8.5(2-10)$ & $8.3(4.5-10.9)$ & \\
\hline Colorectum & $4(2-12.5)$ & $25.5\left(\mathrm{NA}^{\mathrm{b}}\right)$ & \\
\hline Piecemeal resection, $n(\%)$ & $32(86.5)$ & $7(100)$ & NS \\
\hline Average number of pieces & 3.3 & 6.6 & NS \\
\hline
\end{tabular}

$E M R$ endoscopic mucosal resection, $D B$ delayed bleeding, $I Q R$ interquartile range, $N S$ not significant, Vit $K$ antagonists vitamin $\mathrm{K}$ antagonists, DOAC/NOAC direct oral anticoagulants/new oral anticoagulants

${ }^{a}$ Combination therapy defined as concurrent use of aspirin and acenocoumarin

${ }^{\mathrm{b}} N A$ not applicable 
to the EMR and resumed it $48 \mathrm{~h}$ after the procedure. This patient presented with a severe DB 11 days after the EMR of the esophagus. Another patient with familial adenomatous polyposis discontinued antiplatelet therapy on the day of the procedure. He was admitted the day after the EMR with a moderately severe DB. The only patient who used a vitamin $\mathrm{K}$ antagonist (acenocoumarin) stopped the medication three days prior to the EMR of the rectum. At the day of the procedure, the International Normalized Ratio (INR) was 1.1. Five days after the EMR, the antithrombotic medication was started again and he presented with a moderately severe DB 11 days after the EMR with an INR of 3.6.

\section{Discussion}

This prospective pilot study confirms that prophylactic SAP application after EMR is feasible and safe and can be done in a few minutes without significantly delaying the procedure. Larger studies should be performed to determine whether SAP application is indeed effective for the prevention of DB after EMR.

We found that SAP is easy in use. A dose of $3 \mathrm{cc}$ was generally sufficient to fully cover the post-EMR defect. This is comparable to the $2.56 \mathrm{~mL}$ that was previously reported for complete coverage of the defect after endoscopic polyp resection [14]. As the gel is transparent, a clear vision of the underlying defect plane remains possible. Nonetheless, this transparency sometimes also made it difficult to determine whether the area was fully covered. A systematic approach is therefore required, and it is advisable to desufflate during and after application for some minutes to prevent the gel from spreading over non-treated areas.

In four cases, the gel was applied in addition to clip placement (results not shown). Our experience is that the gel can easily be applied between and around the clip bases. This makes the gel suitable for potential adjuvant treatment when complete closure cannot be achieved with clips alone, which occurs in roughly one in five patients [11].

No adverse reactions related to SAP use were seen in our cohort. This includes intestinal occlusion and pancreatitis. Both were suggested in previous studies using SAP [12]. However, when one considers GI anatomy as well as the local use of the gel, these potential adverse events seem highly unlikely in relation to SAP application.

DB was seen in seven of 44 patients (15.9\%), particularly in the duodenum. Although this seems relatively high, our results are in line with DB rates up to $28 \%$ for duodenal and $7-12 \%$ for colorectal lesions reported in the literature $[5,6$, 11]. The DB rate of $6 \%$ after esophageal EMR seems high, compared to previously reported DB rates in the esophagus after EMR up to 2\% [1-3]. Nonetheless, the esophageal DB rate in our cohort can be explained by the fact that the number of treated patients with a lesion in the esophagus was small. We expect that DB rates after EMR of both esophageal and duodenal lesions will likely be lower when more patients are included.

Nonetheless, the DB rate in our study is different from those seen in previous reports in which SAP was applied, which may in part be due to different definitions for DB and inclusion criteria, reflecting different study aims [12-14]. For example, where Subramanian et al. primarily looked into the efficacy of SAP for treatment of intraprocedural bleedings, and studied the DB rates secondary to that, we applied SAP prophylactically. Another explanation for the high DB rate could be a shift in the type of lesions referred, with more complex patients being referred to our tertiary referral center. In view of this, we also critically evaluated risk factors for DB that could potentially explain the relatively high DB rate in this cohort, reflecting a high a priori risk of DB, i.e. antithrombotic medication use, and lesion size and location. Relatively more patients with DB than without were using platelet inhibitors at the time of the EMR. Although aspirin can be continued during endoscopic interventions according to current guidelines, aspirin has been shown to increase the risk of bleeding after EMR procedures [6, 22]. Although a larger lesion size was a prerequisite for inclusion in the study, the mean size was even larger in patients developing DB (see Table 3). Various studies in the colorectum have shown that lesions $\geq 20 \mathrm{~mm}$ have an increased DB risk, and this risk further increases with size [21, 22]. Therefore, we believe that the high DB rate is in line with the high a priori risk of DB in these patients.

Lastly, the majority of the DBs in our cohort were observed in the duodenum (23\%), which is known to be associated with an increased risk of post-EMR bleedings. Compared to the other papers on SAP, where only $15 \%$ and $10 \%$ of the EMRs were performed in the duodenum, this may also be responsible for a higher risk of DB in our cohort compared to those cohorts $[12,14]$.

A strength of the study is that we included both upper and lower GI lesions, which gives insight in the use of SAP at different sites in the GI tract. Furthermore, all included lesions can be considered to have a relatively high risk of DB, as we only included larger lesions [6, 20, 22]. To our knowledge, the largest reported EMR cohort with prophylactic SAP application included 21 patients [14]. In our study, more than double this number of patients were included. In addition to studying feasibility and overall safety, we also collected data on the occurrence, timing and severity of DB. We evaluated DB rates per location in the GI tract, rather than discuss all lesions as a heterogeneous group.

This study also has some limitations as it was performed in a single center, with no control group, and the number of included patients was relatively small not allowing definite conclusions about the potential for prevention of DB. 
Furthermore, we used a qualitative endpoint for the feasibility endpoint, i.e. whether SAP application was considered easy by the endoscopist. In this study, we were unable to determine the inter-observer variability for ease of SAP application. Nonetheless, we did quantify it in terms of application time.

In conclusion, SAP in daily practice is feasible and safe and no clear arguments against the hypothesis that SAP may prevent delayed bleeding after EMR were found. Further studies, preferably comparing SAP with other preventive measures in high-risk populations and requiring a larger sample size, are needed to show its efficacy.

Author contributions ES and AT designed and performed the study, collected, analysed and interpreted the data and wrote the paper. EG and professor PS contributed to the conception and design of the study, interpretation of the data and critically reviewing the manuscript. All authors gave final approval of the version to be published.

Funding The Self-Assembling Peptide in this study was provided without charge by PuraStat ${ }^{\circledR}$ (3-D Matrix Europe, France).

\section{Compliance with ethical standards}

Disclosures Dr. Siersema currently receives research support from Pentax (Japan) and is on the advisory board of Boston Scientific (USA). Dr. van Geenen receives material research support from Olympus (Japan). The authors have no other relevant affiliations or financial involvement with any organization or entity with a financial interest in or financial conflict with the subject matter or materials discussed in the manuscript apart from those disclosed. Dr. Elsa Soons and Dr. Ayla Turan have no conflicts of interest or financial ties to disclose.

Open Access This article is licensed under a Creative Commons Attribution 4.0 International License, which permits use, sharing, adaptation, distribution and reproduction in any medium or format, as long as you give appropriate credit to the original author(s) and the source, provide a link to the Creative Commons licence, and indicate if changes were made. The images or other third party material in this article are included in the article's Creative Commons licence, unless indicated otherwise in a credit line to the material. If material is not included in the article's Creative Commons licence and your intended use is not permitted by statutory regulation or exceeds the permitted use, you will need to obtain permission directly from the copyright holder. To view a copy of this licence, visit http://creativecommons.org/licenses/by/4.0/.

\section{References}

1. Komeda Y, Bruno M, Koch A (2014) EMR is not inferior to ESD for early Barrett's and EGJ neoplasia: an extensive review on outcome, recurrence and complication rates. Endosc Int Open 2(2):E58-64

2. Alvarez Herrero L, Pouw RE, van Vilsteren FG, ten Kate FJ, Visser M, Seldenrijk CA et al (2011) Safety and efficacy of multiband mucosectomy in 1060 resections in Barrett's esophagus. Endoscopy 43(3):177-183

3. Tomizawa Y, Iyer PG, Song LM, Buttar NS, Lutzke LS, Wang KK (2013) Safety of endoscopic mucosal resection for Barrett's esophagus. Am J Gastroenterol 108(9):1440-1447 (quiz 8)
4. Hoteya S, Furuhata T, Takahito T, Fukuma Y, Suzuki Y, Kikuchi D et al (2017) Endoscopic submucosal dissection and endoscopic mucosal resection for non-ampullary superficial duodenal tumor. Digestion 95(1):36-42

5. Yamasaki Y, Uedo N, Takeuchi Y, Ishihara R, Okada H, Iishi H (2018) Current status of endoscopic resection for superficial nonampullary duodenal epithelial tumors. Digestion 97(1):45-51

6. Metz AJ, Bourke MJ, Moss A, Williams SJ, Swan MP, Byth K (2011) Factors that predict bleeding following endoscopic mucosal resection of large colonic lesions. Endoscopy 43(6):506-511

7. Libanio D, Pimentel-Nunes P, Dinis-Ribeiro M (2016) Complications of endoscopic resection techniques for upper GI tract lesions. Best Pract Res Clin Gastroenterol 30(5):735-748

8. Marques J, Baldaque-Silva F, Pereira P, Arnelo U, Yahagi N, Macedo G (2015) Endoscopic mucosal resection and endoscopic submucosal dissection in the treatment of sporadic nonampullary duodenal adenomatous polyps. World J Gastrointest Endosc 7(7):720-727

9. Pohl H, Grimm IS, Moyer MT, Hasan MK, Pleskow D, Elmunzer BJ et al (2019) Clip closure prevents bleeding after endoscopic resection of large colon polyps in a randomized trial. Gastroenterology 157(4):977-84.e3

10. Feagins LA, Smith AD, Kim D, Halai A, Duttala S, Chebaa B et al (2019) Efficacy of prophylactic hemoclips in prevention of delayed post-polypectomy bleeding in patients with large colonic polyps. Gastroenterology 157(4):967-76.e1

11. Albeniz E, Alvarez MA, Espinos JC, Nogales O, Guarner C, Alonso $P$ et al (2019) Clip closure after resection of large colorectal lesions with substantial risk of bleeding. Gastroenterology 57(5):1213-1221

12. Pioche M, Camus M, Rivory J, Leblanc S, Lienhart I, Barret M et al (2016) A self-assembling matrix-forming gel can be easily and safely applied to prevent delayed bleeding after endoscopic resections. Endosc Int Open 4(4):E415-E419

13. Uraoka T, Ochiai Y, Fujimoto A, Goto O, Kawahara Y, Kobayashi $\mathrm{N}$ et al (2016) A novel fully synthetic and self-assembled peptide solution for endoscopic submucosal dissection-induced ulcer in the stomach. Gastrointest Endosc 83(6):1259-1264

14. Subramaniam S, Kandiah K, Thayalasekaran S, Longcroft-Wheaton G, Bhandari P (2019) Haemostasis and prevention of bleeding related to ER: The role of a novel self-assembling peptide. United Eur Gastroenterol J 7(1):155-162

15. Tie J, Wang Y, Tomasetti C, Li L, Springer S, Kinde I et al (2016) Circulating tumor DNA analysis detects minimal residual disease and predicts recurrence in patients with stage II colon cancer. Sci Transl Med 8(346):346ra92

16. Ferlitsch M, Moss A, Hassan C, Bhandari P, Dumonceau JM, Paspatis $\mathrm{G}$ et al (2017) Colorectal polypectomy and endoscopic mucosal resection (EMR): European Society of Gastrointestinal Endoscopy (ESGE) clinical guideline. Endoscopy 49(3):270-297

17. Pouw RE, van Vilsteren FG, Peters FP, Alvarez Herrero L, Ten Kate FJ, Visser M et al (2011) Randomized trial on endoscopic resectioncap versus multiband mucosectomy for piecemeal endoscopic resection of early Barrett's neoplasia. Gastrointest Endosc 74(1):35-43

18. Klein A, Nayyar D, Bahin FF, Qi Z, Lee E, Williams SJ et al (2016) Endoscopic mucosal resection of large and giant lateral spreading lesions of the duodenum: success, adverse events, and long-term outcomes. Gastrointest Endosc 84(4):688-696

19. Cotton PB, Eisen GM, Aabakken L, Baron TH, Hutter MM, Jacobson BC et al (2010) A lexicon for endoscopic adverse events: report of an ASGE workshop. Gastrointest Endosc 71(3):446-454

20. Burgess NG, Metz AJ, Williams SJ, Singh R, Tam W, Hourigan LF et al (2014) Risk factors for intraprocedural and clinically significant delayed bleeding after wide-field endoscopic mucosal resection of large colonic lesions. Clin Gastroenterol Hepatol. 12(4):651-661

21. Jaruvongvanich V, Prasitlumkum N, Assavapongpaiboon B, Suchartlikitwong S, Sanguankeo A, Upala S (2017) Risk factors for 
delayed colonic post-polypectomy bleeding: a systematic review and meta-analysis. Int J Colorectal Dis 32(10):1399-1406

22. Albeniz E, Fraile M, Ibanez B, Alonso-Aguirre P, Martinez-Ares D, Soto $\mathrm{S}$ et al (2016) A scoring system to determine risk of delayed bleeding after endoscopic mucosal resection of large colorectal lesions. Clin Gastroenterol Hepatol 14(8):1140-1147
Publisher's Note Springer Nature remains neutral with regard to jurisdictional claims in published maps and institutional affiliations. 